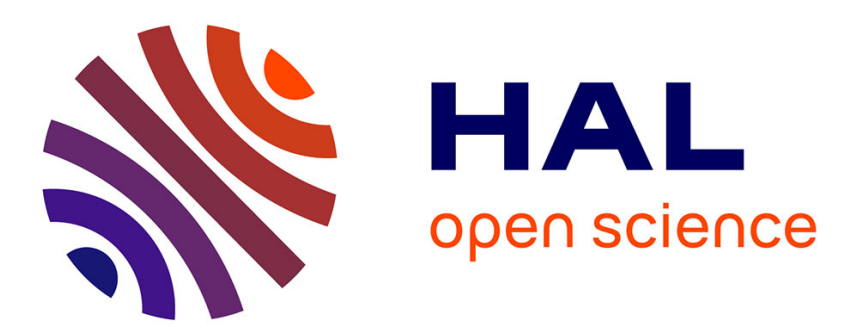

\title{
An Approach to Interval Observers for Takagi-Sugeno Systems with Attractiveness Guarantees
}

Hiroshi Ito, Thach Ngoc Dinh

\section{To cite this version:}

Hiroshi Ito, Thach Ngoc Dinh. An Approach to Interval Observers for Takagi-Sugeno Systems with Attractiveness Guarantees. 2019 58th Annual Conference of the Society of Instrument and Control Engineers of Japan (SICE), Sep 2019, Hiroshima, Japan. pp.1268-1273, 10.23919/SICE.2019.8859956 . hal-02434059

\section{HAL Id: hal-02434059 https://hal-cnam.archives-ouvertes.fr/hal-02434059}

Submitted on 19 Jan 2020

HAL is a multi-disciplinary open access archive for the deposit and dissemination of scientific research documents, whether they are published or not. The documents may come from teaching and research institutions in France or abroad, or from public or private research centers.
L'archive ouverte pluridisciplinaire HAL, est destinée au dépôt et à la diffusion de documents scientifiques de niveau recherche, publiés ou non, émanant des établissements d'enseignement et de recherche français ou étrangers, des laboratoires publics ou privés. 


\title{
An Approach to Interval Observers for Takagi-Sugeno Systems with Attractiveness Guarantees
}

\author{
Hiroshi Ito ${ }^{1 \dagger}$ and Thach Ngoc Dinh ${ }^{2}$ \\ ${ }^{1}$ Department of Intelligent and Control Systems, Kyushu Inst. Technology, Japan \\ (Tel: +81-928-29-7717; E-mail: hiroshi@palm.ces.kyutech.ac.jp) \\ ${ }^{2}$ CNAM, Cedric-Lab, 292 rue St-Martin, 75141 Paris Cedex 03, France \\ (Tel: +33-140-27-2590; E-mail: ngoc-thach.dinh@lecnam.net)
}

\begin{abstract}
This paper proposes a method to design interval observers for nonlinear systems by making use of the TakagiSugeno fuzzy model. The Takagi-Sugeno model has properties which general nonlinear systems do not posses. This paper demonstrates how such properties allow interval observers to handle nonlinearities in unmeasured variables. Instead of computing some intervals only, this paper puts emphasis on clarifying guarantees the observer can provide for a reasonable length of computed intervals in terms of convergence and attractiveness.
\end{abstract}

Keywords: Interval observers; Takagi-Sugeno fuzzy model; Polytypic systems; State estimation.

\section{INTRODUCTION}

Interval observers are practical for estimating the transition of state variables of dynamical systems since they produces time-varying intervals in which the state variables are guaranteed to stay all times $[7,9,17,20]$. Classical observers do not give such guarantees, although they guarantee asymptotically perfect estimate. Broadening the class of systems for which interval observers can be designed is undoubtedly instrumental. As in the case of classical observers, nonlinearities often hampers and sometimes prohibit construction of the interval observers.

The Takagi-Sugeno fuzzy (T-S) model is one of popular models in control engineering [19], and there is strong demand for the use of the T-S model in dealing nonlinearities in applications. It smoothly interpolates multiple linear models which can be handled by basic knowledge. All nonlinearity are condensed into membership functions which weight individual "local" linear models. The T-S model suggests applying linear tools and components with appropriate scheduling. However, finding appropriate scheduling with guarantees is not easy when the transition between the local models are endogenously determined, which actually means nonlinearity of the system. Therefore, designs based on the T-S fuzzy model naturally become technical and complicated by combinations of tools and components which were originally simple. On the other hand, the language of general nonlinear systems can explains and expresses solutions in a succinct way $[8,10,16,21]$. However it literally needs the broadest variety of tools, components and knowledge. Hence, the T-S model is an important target of practical importance in control.

The T-S model exhibits properties which are not enjoyed by general nonlinear systems. This paper aims to demonstrate utilization of such properties in designing interval observers. This paper also pursues the utility of intervals in dealing with nonlinearities associated with unmeasured variables. Tools and component ideas are

$\dagger$ Hiroshi Ito is the presenter of this paper. not necessarily novel. There have been many studies on interval observers for particular classes of systems [3-5, 12-15]. Some attempts have also been made for the T-S type systems $[2,11]$. This paper clarifies how they can be tailored and combined for qualifying strong guarantees to be stated for the T-S model. Usually, it is not very difficult to achieve the framer property, which is the notion of providing intervals in which state variable stay, if one does not care the length of estimated intervals. In fact, one can use an artificial system which over-bounds terms to secure the positivity (non-negativity, more precisely) ensuring the framer property. Therefore, the goal of this paper is to present some reasonable guarantees of the intervals, which is not always clear. Such issues does not seem to have always been paid attention carefully in the literature of interval observers. For this purpose, this paper focuses on making use of the strong property that global behavior of the T-S model is at most linear. In this paper, it is shown that input-to-state stability (ISS) can play an important role in deriving guarantees of convergence and attractiveness which characterize the usefulness of the computed intervals. It should be remarked that independently of methods, intervals can be improved by taking the intersection of intervals produced by a bundle of interval observers [1]. However, this aggregation does not make an increasing interval decrease, and it does not change asymptotic properties either. This explains the importance of guarantees of convergence and attractiveness this paper pursues.

Notation: The symbol $\mathbb{R}$ denotes the set of real numbers. The set of non-negative real numbers is denoted by $\mathbb{R}_{+}$, i.e., $\mathbb{R}_{+}:=[0, \infty)$. A square matrix $M \in \mathbb{R}^{n \times n}$ is said to be positive definite and written as $M \succ 0$ if $v^{\top} M v>0$ holds for all $v \in \mathbb{R}^{n} \backslash\{0\}$. The symbol $|\cdot|$ denotes Euclidean norm of vectors of any dimension. Inequalities with symbols $\leq$ and $\geq$ must be understood componentwise, i.e., for $x_{a}=\left[x_{a, 1}, \ldots, x_{a, n}\right]^{\top} \in \mathbb{R}^{n}$ and $x_{b}=$ $\left[x_{b, 1}, \ldots, x_{b, n}\right]^{\top} \in \mathbb{R}^{n}, x_{a} \leq x_{b}$ if and only if, for all $i \in$ $\{1, \ldots, n\}, x_{a, i} \leq x_{b, i}$. For simplicity, $\left[x_{a}, x_{b}\right]$ denotes the closed set $\left\{x \in \mathbb{R}^{n}: x_{a} \leq x \leq x_{b}\right\}$. A square matrix 
$M \in \mathbb{R}^{n \times n}$ is said to be Metzler if each off-diagonal entry of this matrix is nonnegative. The symbol $\sigma_{\max }(\cdot)$ denotes the largest singular value of a matrix.

\section{A T-S MODEL AND AN INTERVAL OBSERVER}

Consider the following system of in the form of the Takagi-Sugeno Fuzzy model (T-S model for short) accompanied by an output equation

$\dot{x}(t)=\sum_{k=1}^{m} \mu_{k}(x(t)) A_{k} x(t)$

$y(t)=C x(t)$,

where $x(t) \in \mathbb{R}^{n}$ and $y(t) \in \mathbb{R}^{q}$ are the state and the output vectors, respectively. The membership functions $\mu_{k}: \mathbb{R}^{n} \rightarrow[0,1]$ are locally Lipschitz and satisfy

$\sum_{k=1}^{m} \mu_{k}(x)=1, \quad \forall x \in \mathbb{R}^{n}$

System (1a) is assumed to be forward complete. This is assumed to write properties of state estimation for the infinite time horizon. The forward complete assumption is not needed if one stops evaluating properties at the finite escape time of maximal solutions. The model (1a) can also be referred to as a polytypic system since the vertices $\left\{A_{k}\right\}_{i=1}^{m}$ define the trace of $\sum_{k=1}^{m} \mu_{k} A_{k}$ with the variation of $\mu_{k}$ as a polytope on the space of $n \times n$ matrices. If $\mu_{k} \mathrm{~s}$ were exogenous, i.e., $\mu_{k}(t)$ instead of $\mu_{k}(x(t)), \mu_{k} \mathrm{~s}$ could be regarded as scheduling parameters. Since $\mu_{k}(x(t))$ is used in (1a), the T-S model defines a class of nonlinear systems. The system (1a) is said to be non-negative if there is no instant $t \in \mathbb{R}+$ such that $x(t) \nsupseteq 0$ for all initial conditions restricted to $x(0) \in \mathbb{R}_{+}^{n}$. In this paper, whenever the system (1a) is said to be non-negative, the restriction $x(0) \in \mathbb{R}_{+}^{n}$ is assumed $^{1}$. The non-negativity holds if and only if for each $i \in\{1,2, \ldots, n\}$, the implication

$x_{i}=0 \Rightarrow \sum_{k=1}^{m} \mu_{k}(x)\left[A_{k}\right]_{i} x \geq 0$

holds true for all $x \in \mathbb{R}_{+}^{n}$. Here $\left[A_{k}\right]_{i}$ denotes the $i$-th row of the matrix $A_{k} . x_{i}$ denotes the $i$-th component of the vector $x$. Systems in science and engineering are often positive. For instance, systems are non-negative if their state variables are chosen as mass, i.e., energy quantities. The problem this paper addresses is to estimate $x(t)$ which is not measured. The output $y(t)$ is measured. We are interested in not only asymptotic estimation of $x(t)$, but also estimation of time-varying intervals in which all the variables $x_{i}(t)$ stay all times.

This paper proposes the following pair of systems as

${ }^{1}$ The functions $\mu_{k}$ are required to be defined only on $\mathbb{R}_{+}^{n}$ such an interval observer:

$$
\begin{aligned}
\dot{\bar{x}}(t)=\left(A_{0}(\underline{x}, \bar{x})+\right. & \bar{\Delta}(\underline{x}, \bar{x})) \bar{x}(t) \\
& -\bar{L}(\underline{x}, \bar{x})(y(t)-C \bar{x}(t)) \\
\underline{\dot{x}}(t)=\left(A_{0}(\underline{x}, \bar{x})+\right. & \underline{\Delta}(\underline{x}, \bar{x})) \underline{x}(t) \\
& -\underline{L}(\underline{x}, \bar{x})(y(t)-C \underline{x}(t)),
\end{aligned}
$$

where the matrix $\underline{A}(\underline{x}, \bar{x}) \in \mathbb{R}^{n \times n}$ is defined by its $(i, j)$ th component as

$$
\begin{aligned}
{\left[A_{0}\right]_{i, j}(\underline{x}, \bar{x})=} & \frac{1}{2}\left(\overline{\max }_{s \in[\underline{x}, \bar{x}]} \sum_{k=1}^{m} \mu_{k}(s)\left[A_{k}\right]_{i, j}\right. \\
& \left.+\overline{\min }_{s \in[\underline{x}, \bar{x}]} \sum_{k=1}^{m} \mu_{k}(s)\left[A_{k}\right]_{i, j}\right)
\end{aligned}
$$

for $i, j=1,2, \ldots, n$. This paper uses the following operators:

$$
\begin{aligned}
& \overline{\max }_{s \in[\underline{x}, \bar{x}]} f(s)= \begin{cases}\max _{s \in[\underline{x}, \bar{x}]} f(s) & \text { if } \underline{x} \leq \bar{x} \\
f(\bar{x}) & \text { otherwise }\end{cases} \\
& \overline{\min }_{s \in[\underline{x}, \bar{x}]} f(s)= \begin{cases}\min _{s \in[\underline{x}, \bar{x}]} f(s) & \text { if } \underline{x} \leq \bar{x} \\
f(\bar{x}) & \text { otherwise }\end{cases}
\end{aligned}
$$

The matrices $[\bar{\Delta}]_{i, j}(\underline{x}, \bar{x}) \in \mathbb{R}^{n \times n}$ and $[\underline{\Delta}]_{i, j}(\underline{x}, \bar{x}) \in$ $\mathbb{R}^{n \times n}$ in (4) are defined by

$$
[\bar{\Delta}]_{i, j}(\underline{x}, \bar{x})=\overline{\max }_{s \in[\underline{x}, \bar{x}]} \sum_{k=1}^{m} \mu_{k}(s)\left[A_{k}\right]_{i, j}-\left[A_{0}\right]_{i, j}(\underline{x}, \bar{x})
$$

$[\underline{\Delta}]_{i, j}(\underline{x}, \bar{x})=\overline{\min }_{s \in[\underline{x}, \bar{x}]} \sum_{k=1}^{m} \mu_{k}(s)\left[A_{k}\right]_{i, j}-\left[A_{0}\right]_{i, j}(\underline{x}, \bar{x})$

for $i, j=1,2, \ldots, n$. It will be shown that $\bar{x}(t)$ and $\underline{x}(t)$ give an upper bound and a lower bound, respectively, as well as asymptotic estimates of the state $x(t)$ under an appropriate assumption. Both systems (4a) and (4b) are natural extension of the Luenberger observer which is common for nonlinear systems $[16,21]$, except that the system coefficient depends on $\bar{x}$ and $\underline{x}$. It is important to notice that the proposed observer (4) allows the nonlinearities of the target system to depend on the unmeasured state $x$, as in (1a). Since $x$ is not measurable, the pair $\bar{x}$ and $\underline{x}$ are employed by the observer (4). In accordance with these matrices, this paper chooses the observer gains $\bar{L}$ and $\underline{L}$ as

$$
\begin{aligned}
& {[\bar{L}]_{i, j}(\underline{x}, \bar{x})=\sum_{k=1}^{m} \mu_{k}\left(\bar{\zeta}_{i, j}(\underline{x}, \bar{x})\right) \bar{L}_{k}} \\
& {[\underline{L}]_{i, j}(\underline{x}, \bar{x})=\sum_{k=1}^{m} \mu_{k}\left(\underline{\zeta}_{i, j}(\underline{x}, \bar{x})\right) \underline{L}_{k},}
\end{aligned}
$$

where

$$
\begin{aligned}
& \bar{\zeta}_{i, j}(\underline{x}, \bar{x})=\arg \overline{\max }_{s \in[\underline{x}, \bar{x}]} \sum_{k=1}^{m} \mu_{k}(s)\left[A_{k}\right]_{i, j} \\
& \underline{\zeta}_{i, j}(\underline{x}, \bar{x})=\arg \overline{\min }_{s \in[\underline{x}, \bar{x}]} \sum_{k=1}^{m} \mu_{k}(s)\left[A_{k}\right]_{i, j} .
\end{aligned}
$$


For the observer candidate (4) with the above parameters, we can prove the following theorems.

Theorem 1: Suppose that there exist $\bar{P} \succ 0$ and $\underline{P} \succ$ 0 satisfying

$\bar{P}\left(A_{k}+\bar{L}_{k} C\right)+\left(A_{k}+\bar{L}_{k} C\right)^{T} \bar{P} \prec 0$

$\underline{P}\left(A_{k}+\underline{L}_{k} C\right)+\left(A_{k}+\underline{L}_{k} C\right)^{T} \underline{P} \prec 0$

for all $k=1,2 \ldots, m$. Then the implication

$\lim _{t \rightarrow \infty} x(t)=0 \Rightarrow \lim _{t \rightarrow \infty} \bar{e}(t)=\lim _{t \rightarrow \infty} \underline{e}(t)=0$

hold true of all $x(0), \bar{x}(0), \underline{x}(0) \in \mathbb{R}^{n}$, and there exists $g \geq 0$ such that

$\limsup _{t \rightarrow \infty}|\bar{x}(t)-\underline{x}(t)| \leq g \limsup _{t \rightarrow \infty}|x(t)|$

holds of all $x(0), \bar{x}(0), \underline{x}(0) \in \mathbb{R}^{n}$. Furthermore, in the case of $\bar{L}_{k}=\underline{L}_{k}, k=1,2 \ldots, m$, the implication

$\underline{x}\left(t_{s}\right)=\bar{x}\left(t_{s}\right) \Rightarrow \forall t \in\left[t_{s}, \infty\right) \quad \underline{x}(t)=\bar{x}(t)$

holds true of all $x(0), \bar{x}(0), \underline{x}(0) \in \mathbb{R}^{n}$.

Theorem 2: Suppose that system (1a) is nonnegative. If the matrices $A_{k}+\bar{L}_{k} C$ and $A_{k}+\underline{L}_{k} C$ are Metzler for all $k=1,2 \ldots, m$, then

$\forall t \in \mathbb{R}_{+} \quad \underline{x}(t) \leq x(t) \leq \bar{x}(t)$

holds true for all $x(0), \bar{x}(0), \underline{x}(0) \in \mathbb{R}_{+}^{n}$ satisfying

$\underline{x}(0) \leq x(0) \leq \bar{x}(0)$.

Property (14) in Theorem 2 is referred to as the framer property in the literature. It ensures that $x(t)$ is bounded from above and below by $\bar{x}(t)$ and $\underline{x}(t)$, respectively, all times. However, if the lengths $\bar{x}_{i}(t)-\underline{x}_{i}(t)$ of the interval are very large or rapidly increasing with time, the interval is useless. Hence, the properties in Theorem 1 are sought in this paper. If one removes the cause clause $\lim _{t \rightarrow \infty} x(t)=0$, property (11) is the standard observer property. Since the T-S model (1a) is nonlinear in unmeasured variables, guaranteeing complete elimination of estimation error regardless of non-zero unmeasured variables is not obvious as is known in the standard problem of designing observer for general nonlinear systems [16, 21]. In this sense, properties (11) and (12) established in Theorem 1 are reasonable. Property (12) is referred to as global ultimate boundedness, and the constant $g$ is called the asymptotic gain in the field of nonlinear control theory. The achievement of (11) and (12) is due to input-to-state stability (ISS) regarding $x$ as the input as shown in the next section. Property (13) demonstrates that the agreement $\bar{x}(t)=x(t)$ is an equilibrium regardless of the variation of $x(t)$. Therefore, the attractiveness of the agreement guaranteed in this paper is stronger than just having $\lim _{t \rightarrow \infty} \bar{x}(t)=\lim _{t \rightarrow \infty} \underline{x}(t)$ implied by (11) under the assumption of $\lim _{t \rightarrow \infty} x(t)=0$.

Remark 1: If there exists a locally Lipschitz function $\hat{\mu}_{k}$ such that

$\mu_{k}(x)=\hat{\mu}_{k}(y), \quad \forall x \in \mathbb{R}^{n}$ for all $k=1,2, \ldots, m$, the membership functions are said to be measurable. In this measurable case, it is straightforward to see that the pair of the systems

$$
\begin{aligned}
& \dot{\bar{x}}(t)=\sum_{k=1}^{m} \hat{\mu}_{k}(y(t)) A_{k} \bar{x}(t)-\bar{L}(y(t))(y(t)-C \bar{x}(t)) \\
& \underline{\dot{x}}(t)=\sum_{k=1}^{m} \hat{\mu}_{k}(y(t)) A_{i} \underline{x}(t)-\underline{L}(y(t))(y(t)-C \underline{x}(t))
\end{aligned}
$$

with

$$
\begin{aligned}
& \bar{L}(y(t))=\sum_{k=1}^{m} \hat{\mu}_{k}(y(t)) \bar{L}_{k} \\
& \underline{L}(y(t))=\sum_{k=1}^{m} \hat{\mu}_{k}(y(t)) \underline{L}_{k}
\end{aligned}
$$

yields

$$
\begin{aligned}
& \dot{\bar{e}}(t)=\sum_{k=1}^{m} \hat{\mu}_{k}(y)\left(A_{i}+\bar{L}_{i} C\right) \bar{e}(t) \\
& \dot{e}(t)=\sum_{k=1}^{m} \hat{\mu}_{k}(y)\left(A_{i}+\underline{L}_{i} C\right) \underline{e}(t) .
\end{aligned}
$$

Therefore, all the statements of Theorems 1 and 2 hold true without the cause clause $\lim _{t \rightarrow \infty} x(t)=0$ in (11).

\section{PROOF OF THEOREMS 1 AND 2}

Define $\bar{e}=\bar{x}-x$ and $\underline{e}=x-\underline{x}$. From (1a) and (4) we obtain

$$
\begin{aligned}
\dot{\bar{e}}=\left(A_{0}(\underline{x}, \bar{x})+\bar{\Delta}(\underline{x}, \bar{x})+\right. & \bar{L}(\underline{x}, \bar{x}) C) \bar{e} \\
& +\bar{R}(x, \underline{x}, \bar{x}) x \\
\underline{\dot{e}}=\left(A_{0}(\underline{x}, \bar{x})+\underline{\Delta}(\underline{x}, \bar{x})\right) & +\underline{L}(\underline{x}, \bar{x}) C) \underline{e} \\
& +\underline{R}(x, \underline{x}, \bar{x}) x,
\end{aligned}
$$

where

$\bar{R}(x, \underline{x}, \bar{x})=A_{0}(\underline{x}, \bar{x})+\bar{\Delta}(\underline{x}, \bar{x})-\sum_{k=1}^{m} \mu_{k}(x) A_{k}$
$\underline{R}(x, \underline{x}, \bar{x})=\sum_{k=1}^{m} \mu_{k}(x) A_{k}-A_{0}(\underline{x}, \bar{x})-\underline{\Delta}(\underline{x}, \bar{x})$.

Due to the definitions (5) and (6), we have

$$
\begin{aligned}
\left.A_{0}(\underline{x}, \bar{x})+\underline{\Delta}(\underline{x}, \bar{x})\right) & \leq \sum_{k=1}^{m} \mu_{k}(x) A_{k} \\
& \left.\leq A_{0}(\underline{x}, \bar{x})+\bar{\Delta}(\underline{x}, \bar{x})\right)
\end{aligned}
$$

for all $x, \bar{x}, \underline{x} \in \mathbb{R}_{+}^{n}$ satisfying $\underline{x} \leq x \leq \bar{x}$. It is also verified that

$$
\begin{aligned}
& A_{0}(x, x)=\sum_{k=1}^{m} \mu_{k}(x) A_{k}, \quad \forall x \in \mathbb{R}_{+} \\
& \Delta(x, x)=0, \quad \forall x \in \mathbb{R}_{+} \\
& \bar{\Delta}(x, x)=0, \quad \forall x \in \mathbb{R}_{+} .
\end{aligned}
$$


If one picks $\bar{L}_{k}=\underline{L}_{k}$ for all $k=1,2 \ldots, m$, summing up both sides of (21) and (22) yields

$\frac{d}{d t}(\bar{x}-\underline{x})=0$

for all $x, \bar{x}=\underline{x} \in \mathbb{R}_{+}^{n}$, due to (24), (25) and (26). These are keys to the establishment of Theorems 1 and 2 .

\subsection{Proof of Theorem 1}

By virtue of (5), (6) and (7), the existence of $\bar{P} \succ 0$ satisfying (9) for all $k=1,2 \ldots, m$ guarantees the existence of $\bar{\epsilon}>0$ such that

$$
\begin{aligned}
& \bar{P}\left(A_{0}(\underline{x}, \bar{x})+\bar{\Delta}(\underline{x}, \bar{x})+\bar{L}(\underline{x}, \bar{x}) C\right)+ \\
& \quad\left(A_{0}(\underline{x}, \bar{x})+\bar{\Delta}(\underline{x}, \bar{x})+\bar{L}(\underline{x}, \bar{x}) C\right)^{T} \bar{P}+\bar{\epsilon} \bar{P} \prec 0
\end{aligned}
$$

for all $\bar{x}, \underline{x} \in \mathbb{R}_{+}^{n}$, due to $\mu_{k}(x) \geq 0$ and (2). In the same way, property (10) guarantees the existence of $\underline{\epsilon}>0$ such that

$$
\begin{aligned}
& \underline{P}\left(A_{0}(\underline{x}, \bar{x})+\underline{\Delta}(\underline{x}, \bar{x})+\underline{L}(\underline{x}, \bar{x}) C\right)+ \\
& \quad\left(A_{0}(\underline{x}, \bar{x})+\underline{\Delta}(\underline{x}, \bar{x})+\underline{L}(\underline{x}, \bar{x}) C\right)^{T} \underline{P}+\underline{\epsilon P} \prec 0
\end{aligned}
$$

for all $\bar{x}, \underline{x} \in \mathbb{R}_{+}^{n}$. The function $\bar{V}(\bar{e})=\bar{e}^{T} \bar{P} \bar{e}$ satisfies

$\dot{\bar{V}}(\bar{e}) \leq-(\bar{\epsilon}-\bar{\delta}) \bar{e}^{T} \bar{P} \bar{e}+\frac{1}{\bar{\delta}} x^{T} \bar{R}^{T} \overline{P R} x$

along the solution of (21) for any $\bar{\delta} \in(0, \bar{\epsilon})$. Property (2) implies that all the elements of the matrix $\bar{R}(x, \underline{x}, \bar{x})$ are bounded. Thus, there exists $\bar{c}>0$ such that

$\bar{c} \geq \sigma_{\max }\left(\sup _{x, \bar{x}, \underline{x} \in \mathbb{R}^{n}} \bar{R}(x, \underline{x}, \bar{x})\right)$

Thus, for $\bar{\delta}=\bar{\epsilon} / 2$ we obtain

$\dot{\bar{V}}(\bar{e}) \leq-\frac{\bar{\epsilon}}{2} \bar{p}_{\min }|\bar{e}|^{2}+\frac{2 \bar{c}^{2} \bar{p}_{\max }}{\bar{\epsilon}}|x|^{2}$,

where $\bar{p}_{\max }$ (resp., $\bar{p}_{\min }$ ) is the largest (resp., smallest) eigenvalue of $\bar{P}$. Hence, $\bar{V}(\bar{e})=\bar{e}^{T} \bar{P} \bar{e}$ is an ISS Lyapunov function of system (21) [18]. In the same way, $\underline{V}(\underline{e})=\underline{e}^{T} \underline{P e}$ is an ISS Lyapunov function of system (22). Thereby, the implication (11) follows from the definition of ISS [18]. ISS of (21) also gives

$\limsup _{t \rightarrow \infty}|\bar{e}(t)| \leq \frac{2 \bar{c}}{\bar{\epsilon}} \sqrt{\frac{\bar{p}_{\text {max }}}{\bar{p}_{\text {min }}}} \limsup _{t \rightarrow \infty}|x(t)|$

for all $x(0) \in \mathbb{R}^{n}$. Here, $(2 \bar{c} / \bar{\epsilon}) \sqrt{\bar{p}_{\max } / \bar{p}_{\min }}$ is called the asymptotic gain ${ }^{2}$. Repeat the same argument to obtain the asymptotic gain of (22). With the help of $\bar{x}-\underline{x}=\bar{e}+\underline{e}$, the combination of the two asymptotic gains yields (12) with

$g=\frac{2 \bar{c}}{\bar{\epsilon}} \sqrt{\frac{\bar{p}_{\max }}{\bar{p}_{\min }}}+\frac{2 \underline{c}}{\underline{\epsilon}} \sqrt{\frac{\underline{p}_{\max }}{\underline{p}_{\min }}}$

\footnotetext{
${ }^{2}$ When Euclidean norm is chosen to evaluate the magnitude of the state and the input in the framework of ISS as in (31) and (32), the asymptotic gain is the notion incorporating the effect of initial conditions into the $L_{2}$ - gain [18].
}

Property (13) holds since $\bar{x}=\underline{x}$ implies $d(\bar{x}-\underline{x}) / d t=0$.

Remark 2: The convergence property (11) relies on specific dynamics of the T-S model. The T-S model is nonlinear, but its vector filed grows at most linearly since (2) holds. The linearity allows one to obtain the ISS property (31). The convergence property (11) cannot always be expected for general nonlinear systems.

\subsection{Proof of Theorem 2}

Since $A_{k}+\bar{L}_{k} C$ is Metzler for all $k=1,2 \ldots, m$, by virtue of $\mu_{k}(x) \geq 0$ and (2), (5), (6) and (7), the matrix

$A_{0}(\underline{x}, \bar{x})+\bar{\Delta}(\underline{x}, \bar{x})+\bar{L}(\underline{x}, \bar{x}) C$

is Metzler. In the same way, since $A_{k}+\underline{L}_{k} C$ is Metzler for all $k=1,2 \ldots, m$, the matrix

$A_{0}(\underline{x}, \bar{x})+\underline{\Delta}(\underline{x}, \bar{x})+\underline{L}(\underline{x}, \bar{x}) C$

is Metzler. Suppose that $\bar{x}\left(t_{s}\right)=x\left(t_{s}\right) \geq \underline{x}\left(t_{s}\right)$ holds at some $t \in \mathbb{R}_{+}$. Due to the non-negativity $x(t) \geq 0$ of (1a) and (23), we have

$\bar{R}\left(x\left(t_{s}\right), \underline{x}\left(t_{s}\right), \bar{x}\left(t_{s}\right)\right) x\left(t_{s}\right) \geq 0$
$\underline{R}\left(x\left(t_{s}\right), \underline{x}\left(t_{s}\right), \bar{x}\left(t_{s}\right)\right) x\left(t_{s}\right) \geq 0$.

These properties allow (21) and (22) to yield $\dot{\bar{e}}\left(t_{s}\right) \geq 0$ and $\underline{\dot{e}}\left(t_{s}\right) \geq 0$. These two properties are also verified for $\bar{x}\left(t_{s}\right) \geq x\left(t_{s}\right)=\underline{x}\left(t_{s}\right)$. Since the solutions $x, \bar{x}$ and $\underline{x}$ of (1a), (4a) and (4b) are continuous with respect to $t$, the restriction (15) of the initial condition results in (14).

\section{ASYMPTOTIC GAIN REDUCTION AND FEEDBACK}

The smaller $\bar{x}-\underline{x}=\bar{e}+\underline{e}$ is, the more useful the interval estimate is. Properties (11) and (12) together with (13) and (14) in Theorems 1 and 2 indicate that the reduction of the magnitude of $x$ improve the interval estimate. A narrower interval estimate is obtained by reducing the asymptotic gain of the estimation error system (21) (resp. (22)) from $x$ to $\bar{e}$ (resp. $\underline{e}$ ). According to (31) and (33), the asymptotic gain is guaranteed to be finite, and a smaller asymptotic gain $g$ is achieved in (12) by using larger $\bar{\epsilon}>0$ and $\underline{\epsilon}>0$ in

$$
\begin{aligned}
& \bar{P}\left(A_{k}+\bar{L}_{k} C\right)+\left(A_{k}+\bar{L}_{k} C\right)^{T} \bar{P}+\underline{\epsilon} \bar{P} \prec 0 \\
& \underline{P}\left(A_{k}+\underline{L}_{k} C\right)+\left(A_{k}+\underline{L}_{k} C\right)^{T} \underline{P}+\underline{\epsilon P}, \prec 0
\end{aligned}
$$

which replace (9) and (10). In fact, the existence of $\bar{\epsilon}$ and $\underline{\epsilon}$ for (34) and (35) leads to (28) and (29) for the same $\bar{\epsilon}$ and $\underline{\epsilon}$. It is important to note that the parameters $\bar{\epsilon}$ and $\underline{\epsilon}$ cannot be made arbitrarily small since $\bar{L}_{k}$ and $\bar{L}_{k}$ are restricted to ones achieving the Metzler property required in Theorem 2.

It is possible to answer the question of how small the asymptotic gain needs to be. In fact, we can achieve the convergence of $\bar{e}(t)$ and $\underline{e}(t)$ to zero without assumptions on $x(t)$ if one is able to apply control input appropriately. 
To illustrate this point, the remainder of this section considers the simple feedback system

$$
\begin{aligned}
\dot{x}(t) & =\sum_{k=1}^{m} \mu_{k}(x(t))\left(A_{k} x(t)+B_{k} u(t)\right) \\
y(t) & =C x(t), \\
u(t) & =\bar{F} x(t),
\end{aligned}
$$

where $u(t) \in \mathbb{R}^{r}$ is the input. To deal with the added input term, replace (4) by

$$
\begin{gathered}
\dot{\bar{x}}(t)=\left(A_{0}(\underline{x}, \bar{x})+\bar{\Delta}(\underline{x}, \bar{x})\right) \bar{x}(t)+\bar{D}(\underline{x}, \bar{x}) \bar{x}(t) \\
-\bar{L}(\underline{x}, \bar{x})(y(t)-C \bar{x}(t)) \\
\dot{x}(t)=\left(A_{0}(\underline{x}, \bar{x})+\underline{\Delta}(\underline{x}, \bar{x})\right) \underline{x}(t)+\underline{D}(\underline{x}, \bar{x}) \bar{x}(t) \\
-\underline{L}(\underline{x}, \bar{x})(y(t)-C \underline{x}(t)),
\end{gathered}
$$

where the matrices $\bar{D}(\underline{x}, \bar{x}), \underline{D}(\underline{x}, \bar{x}) \in \mathbb{R}^{n \times r}$ are defined by their $(i, j)$-th components as

$$
\begin{aligned}
& {[\bar{D}]_{i, j}(\underline{x}, \bar{x})=\overline{\max }_{s \in[\underline{x}, \bar{x}]} \sum_{k=1}^{m} \mu_{k}(s)\left[B_{k} \bar{F}\right]_{i, j}} \\
& {[\underline{D}]_{i, j}(\underline{x}, \bar{x})=\overline{\min _{s \in[\underline{x}, \bar{x}]}} \sum_{k=1}^{m} \mu_{k}(s)\left[B_{k} \bar{F}\right]_{i, j} .}
\end{aligned}
$$

For (36), (37) and (38), we can prove the following.

Theorem 3: Suppose that there exist $\bar{P} \succ 0, \underline{P} \succ 0$, $\bar{Q} \succ 0$ and $\bar{\epsilon}>0, \underline{\epsilon}>0, \bar{\omega}>0$ such that (35),

$$
\begin{gathered}
\bar{P}\left(A_{k}+\bar{L}_{k} C+\Delta_{k}\right)+\left(A_{k}-\bar{L}_{k} C+\Delta_{k}\right)^{T} \bar{P} \\
+\bar{\epsilon} \bar{P} \prec 0 \\
\bar{Q}\left(A_{k}+B_{k} \bar{F}\right)+\left(A_{k}+B_{k} \bar{F}\right)^{T} \bar{Q}+\bar{\omega} \bar{Q} \prec 0
\end{gathered}
$$

hold for all $\Delta_{k} \in \mathbb{R}^{n \times n}$ satisfying

$$
0 \leq\left[\Delta_{k}\right]_{i, j} \leq\left|\left[B_{k} \bar{F}\right]_{i, j}\right|, \quad i, j=1,2, \ldots, n
$$

for all $k=1,2 \ldots, m$. Let $b, c \geq \mathbb{R}_{+}$be such that

$$
\begin{aligned}
b \geq \sigma_{\max } & \left(\sup _{x \in \mathbb{R}^{n}} \sum_{k=1}^{m} \mu_{k}(x) B_{k} \bar{F}\right) . \\
c \geq \sigma_{\max } & \left(\sup _{x, \bar{x}, \underline{x} \in \mathbb{R}^{n}} \bar{R}(x, \underline{x}, \bar{x})\right. \\
& \left.+\bar{D}(x, \underline{x}, \bar{x})-\sum_{k=1}^{m} \mu_{k}(x) B_{k} \bar{F}\right)
\end{aligned}
$$

Let $\bar{p}_{\max }$ (resp., $\bar{p}_{\min }$ ) be the largest (resp., smallest) eigenvalue of $\bar{P}$, Let $\bar{q}_{\max }$ (resp., $\bar{q}_{\text {min }}$ ) denote the largest (resp., smallest) eigenvalue of $\bar{Q}$. If

$$
\frac{16 \bar{p}_{\max } \bar{q}_{\max } c^{2} b^{2}}{\bar{p}_{\min } \bar{q}_{\min } \bar{\epsilon}^{2} \bar{\omega}^{2}}<1
$$

holds, then

$$
\lim _{t \rightarrow \infty} \bar{e}(t)=\lim _{t \rightarrow \infty} \underline{e}(t)=0
$$

holds of all $x(0), \bar{x}(0), \underline{x}(0) \in \mathbb{R}^{n}$. Furthermore, in the case of $\bar{L}_{k}=\underline{L}_{k}, k=1,2 \ldots, m$, the implication (13) holds true of all $x(0), \bar{x}(0), \underline{x}(0) \in \mathbb{R}^{n}$.

Theorem 4: Suppose that

$\sum_{k=1}^{m} \mu_{k}(x) B_{k} \bar{F} \geq 0, \quad \forall x \in \mathbb{R}_{+}$.

If $A_{k}+B_{k} \bar{F}, A_{k}+\bar{L}_{k} C$ and $A_{k}+\underline{L}_{k} C$ are Metzler for all $k=1,2 \ldots, m$, then (14) holds for all $x(0), \bar{x}(0)$, $\underline{x}(0) \in \mathbb{R}_{+}^{n}$ satisfying (15).

Theorems 3 and 4 aim at the idea of feedback to achieve stabilization and interval estimation at the same time. Theorems 3 and 4 can be very restrictive. In fact, the feedback gain (36c) is too simple to allow a wide variety of systems to satisfy the conditions required in Theorems 3 and 4. Nevertheless, the mechanism of proving Theorems 3 and 4 is as simple as looking at the interconnection of the following three systems:

$$
\begin{aligned}
\dot{x}= & \sum_{k=1}^{m} \mu_{k}(x)\left(A_{k}+B_{k} \bar{F}\right) x \\
& +\sum_{k=1}^{m} \mu_{k}(x) B_{k} \bar{F} \bar{e} \\
\dot{\bar{e}}= & \left.\left(A_{0}(\underline{x}, \bar{x})+\bar{\Delta}(\underline{x}, \bar{x})\right)+\bar{L}(\underline{x}, \bar{x}) C\right) \bar{e} \\
+ & \bar{R}(x, \underline{x}, \bar{x}) x+\left(\bar{D}(\underline{x}, \bar{x})-\sum_{k=1}^{m} \mu_{k}(x) B_{k} \bar{F}\right) \bar{x} \\
= & \left(A_{0}(\underline{x}, \bar{x})+\bar{\Delta}(\underline{x}, \bar{x})+\bar{L}(\underline{x}, \bar{x}) C\right. \\
& +\left(\bar{R}(\underline{x}, \bar{x})-\sum_{k=1}^{m} \mu_{k}(x) B_{k} \bar{F}\right) \bar{e} \\
& \left.(x, \underline{x}, \bar{x})+\bar{D}(\underline{x}, \bar{x})-\sum_{k=1}^{m} \mu_{k}(x) B_{k} \bar{F}\right) x \\
\underline{\dot{e}} & =\left(A_{0}(\underline{x}, \bar{x})+\underline{\Delta}(\underline{x}, \bar{x})\right)+\underline{L}(\underline{x}, \bar{x}) C \underline{e} \underline{(x}(x, \underline{x}, \bar{x}) x+\left(\sum_{k=1}^{m} \mu_{k}(x) B_{k} \bar{F}-\underline{D}(\underline{x}, \bar{x})\right) \bar{x} \\
& +\underline{R}
\end{aligned}
$$

Based on the argument used in (31) with the help of (39)-(43), it is verified that the small-gain condition (44) allows the existence of $K>0$ such that $V(x, \bar{e})=$ $x^{T} \bar{Q} x+K \bar{e}^{T} \bar{P} \bar{e}$ is a Lyapunov function establishing global asymptotic stability of the equilibrium $(x, \bar{e})=$ $(0,0)$ of the feedback interconnection of (47) and (48). Moreover, $\underline{V}(\underline{e})=\underline{e}^{T} \underline{P e}$ is an ISS Lyapunov function of (49) with respect to the input $(x, \bar{x})$. Note that $(x, \bar{e}) \rightarrow(0,0)$ implies $\bar{x} \rightarrow 0$. Thereby (45) follows from the cascade system where system (49) is driven by the feedback connection of (47) and (48). It can be verified by the definitions (38a) and (38b) that summing up both sides of (48) and (49) yields (27) when $\bar{L}_{k}=\underline{L}_{k}$ are chosen for all $k=1,2 \ldots, m$, Hence, we arrive at (13). Property (46), the definitions (38a), (38b), the Metzler property of $A_{k}+B_{k} \bar{F}, A_{k}+\bar{L}_{k} C$ and $A_{k}+\underline{L}_{k} C$ in (47), (48) and (49) establishes $x(t) \geq 0$ and (14) holds for all $x(0), \bar{x}(0), \underline{x}(0) \in \mathbb{R}_{+}^{n}$ as long as (15). 


\section{CONCLUDING REMARKS}

This paper has focused on proposing the primary idea of developing reasonable interval observers of T-S systems involving nonlinearities with respect to unmeasured variables. By the reasonability, this paper means convergence and attractiveness properties to avoid very wide or exploding intervals estimated by the observers. To achieve this goal, this paper has introduced intervaldependent nonlinearities judiciously to the observers. It has also been demonstrated that the framework of ISS fits the dynamics of the T-S model and allows one to obtain convergence guarantees. Extension allowing process disturbances is possible, and it will be reported in a separate paper. It is worth developing a way to increase practical usefulness of the combined use of interval observers and feedback stabilizers discussed in Section 4.

It should not be hard to modify the proposed observers to obtain the framer property without imposing the nonnegativity on the T-S model. Nevertheless, obtaining some useful convergence and attractiveness guarantees does not seem straightforward. As known in the literature of interval observer design, removing the non-negativity assumption allows one to use coordinate transformation as done often for relaxing the Metzler requirement.

Finally, it would be worth mentioning that evaluating the magnitude of the bounds $\bar{x}$ and $\underline{x}$ seems easier than that of the estimation errors $\bar{x}-x$ and $x-\underline{x}$. For example, in the presence of a process disturbance, the $L_{2}$-gain from the disturbance to $\bar{x}$ and $\underline{x}$ can be evaluated, and one would be able to numerically search observer gains for a smaller $L_{2}$-gain. Useful discussions on $\operatorname{such} L_{2}$-gain reduction are given in [6] for some classes of systems. Such reduction can be effective to avoid rapidly increasing magnitude of $\bar{x}$ and $x$. However, its practical usefulness is limited since it does not pay attention to the actual state $x$. Therefore, this paper has pursued convergence and attractiveness properties of $\bar{x}-x, x-\underline{x}$ and $\bar{x}-\underline{x}$.

\section{ACKNOWLEDGMENT}

The work was supported in part by JSPS KAKENHI Grant Number 17K06499.

\section{REFERENCES}

[1] O. Bernard, J.L. Gouzé. Closed loop observers bundle for uncertain biotechnical models, J. Process Control 14 (7), 765-774. 2004

[2] S. Chebotarev, D. Efimov, T. Raïssi, A. Zolghadri. Interval Observers for Continuous-Time LPV Systems with L1/L2 Performance, Automatica 58, 8289. 2015

[3] T.N. Dinh and H. Ito. Interval Observers for Continuous-time Bilinear Systems with Discretetime Outputs, 15th European Control Conference, pp.1418-1423, 2016

[4] T.N. Dinh, F. Mazenc, S.-I and Niculescu. Interval observer composed of observers for nonlinear sys- tems, 13th European Control Conference, pp.660665, 2014

[5] D. Efimov, T. Raïssi, S. Chebotarev and A. Zolghadri. Interval state observer for nonlinear time varying systems, Automatica, 49 (1), 200-205, 2013

[6] D. Efimov and T. Raïssi. Design of interval observers for uncertain dynamical systems, Automation and Remote Control, 77 (2), 191-225, 2016

[7] J.L. Gouzé, A. Rapaport and M.Z. Hadj-Sadok. Interval observers for uncertain biological systems, Ecological modelling 133 (1-2), 45-56, 2000

[8] A. Isidori. Nonlinear control systems, an introduction, 3 rd. edition, Springer, 2001

[9] H. Ito and T.N. Dinh. Interval observers for nonlinear systems with appropriate output feedback, 2nd SICE Int. Symp. Control Systems, pp.9-14, 2016

[10] H.K. Khalil. Nonlinear Control, Global Edition, Pearson, 2015

[11] J. Li, Z. Wang, Y. Shen and Y. Wang. Interval Observer Design for Discrete-Time Uncertain Takagi-Sugeno Fuzzy Systems, IEEE Transactions on Fuzzy Systems 27 (4), 816-823, 2019

[12] F. Mazenc and O. Bernard. Interval observers for linear time-invariant systems with disturbances, $\mathrm{Au}$ tomatica 47 (1), 140-147, 2011

[13] F. Mazenc and T.N. Dinh. Construction of interval observers for continuous-time systems with discrete measurements, Automatica 50 (10), 2555 2560, 2014

[14] F. Mazenc, T.N. Dinh and S.-I. Niculescu. Robust interval observers and stabilization design for discrete-time systems with input and output, Automatica 49 (11), 3490-3497, 2013

[15] F. Mazenc, T.N. Dinh and S.-I. Niculescu. Interval observers for discrete-time systems, Int. J. Robust and Nonlinear Control. 24 (17), 2867-2890, 2014

[16] H. Nijmeijer and A.J. Van Der Schaft. Nonlinear $d y$ namical control systems, Springer, 1990

[17] T. Raissi, D. Efimov and A. Zolghadri. Interval state estimation for a class of nonlinear systems, IEEE Trans. Autom. Control 57 (1), 260-265, 2012

[18] E.D. Sontag and Y. Wang. On characterizations of input-to-state stability property, Systems \& Contr. Letters 24 (5), 351-359, 1995

[19] T. Takagi and M. Sugeno. Fuzzy identification of systems and its applications to modeling and control, IEEE Trans. Systems, Man, Cybernetics, 15 (1), 116-132, 1985

[20] G. Zheng, D. Efimov and W. Perruquetti. Design of interval observer for a class of uncertain unobservable nonlinear systems, Automatica 63 (1) 167-174, 2016

[21] A. J. Krener and A. Isidori. "Linearization by output injection and nonlinear observers," Syst. Control Lett., 3 (4), pp.47-52, 1983 